\title{
La productividad del cuerpo. Apuntes de una fenomenología política en Judith Butler*
}

\author{
The productivity of the body. \\ Notes of a political phenomenology in Judith Butler
}

JESÚS GONZÁLEZ FISAC**

\begin{abstract}
Resumen: el uso que Butler ha hecho de la fenomenología ha sido constante en su obra y siempre ha tenido cariz político. En los últimos años, al hilo de sus trabajos sobre las ocupaciones del espacio público de la llamada "privamera árabe" (así como sobre sus epifenómenos occidentales, como el movimiento "Occupy Wall Street"), Butler ha revisado el concepto arendtiano de "espacio de aparición”, donde, ciertamente, algo así como una fenomenología política se trasparece totalmente.

Palabras clave: Judith Butler, fenomenología, cuerpo, políticas de la calle.
\end{abstract}

\begin{abstract}
Butler's use of phenomenology has been constant in his work and has always had political overtones. In recent years, in his works on the occupations of public space called "Arab Spring" (as well as on their Western epiphenomena, the movement "Occupy Wall Street"), Butler has reviewed the Arendtian concept of "space of appearance" where, indeed, a political phenomenology is fully trasparent.
\end{abstract}

Keywords: Judith Butler, phenomenology, body, politics of the street.

\section{La fenomenología como herramienta crítica}

La idea básica de toda fenomenología es que lo que hay es lo que se muestra. Grosso modo, la fenomenología sostiene que la inteligibilidad de lo que hay está en su mostrarse y en las condiciones de ese mostrarse, sin que el fenómeno deba remitirse o referirse a una

Fecha de recepción: 24/04/2016. Fecha de aceptación: 15/09/2016.

* Este trabajo fue leído en el VII Congreso de la SAF (Cádiz, 24-27 de mayo de 2015). Desarrolla parte de una conferencia anterior, "Cuerpos y espacio público", leída en el XV International Association Women Philosophers Symposium (Alcalá de Henares, 24-27 de junio de 2014). El trabajo ha sido realizado en el marco del proyecto de investigación "Naturaleza humana y comunidad III: ¿Inactualidad del hombre y actualidad del humanismo?” (ref. FF12013-46815-P), financiado por el Ministerio de Educación de España.

** Universidad de Cádiz, Profesor Asociado, e-mail: jesus.gonzalez@uca.es. Actualmente trabaja cuestiones relativas a la antropología y la filosofía de la ilustarción en Kant, así como acerca de la fenomenología política y las emociones en Judith Butler. Ha publicado "Retórica y fenomenología. Exterioridad y vulnerabilidad en el campo del lenguaje", en Sabsay, L., Soley-Beltran, P. Judith Butler en disputa (Barcelona, Egales, 2012), y "La minoría de edad como despotismo de las facultades. Antropología y política en la Beantwortung", Ideas y Valores, 163 (2017, en prensa). 
instancia sustantiva, que es lo que habitualmente se entiende por "fundamento", cuando se piensa en lo que hay como cosa o como naturaleza, o "causa", cuando se piensa como suceder o como acontecer. Cierta fenomenología ha buscado ese ámbito de mostración en un sujeto originario - pero en cualquier caso accesible- por medio de un procedimiento de reducción, sujeto en el que sería posible una intuición de esencias "(Husserl). Pero Butler rechaza todo intento de poder dar cuenta del fenómeno remitiéndolo a un ámbito que, aunque no se muestre inmediatamente, está pertinazmente presente. Butler, vamos a decirlo así, rechaza de plano cualquier esencia. El fenómeno tiene que sostenerse a sí mismo y desde su propio ámbito de mostración, que es el que tiene que ser inmediato y no puede apuntar a otro lugar. Butler critica por tanto la noción de sujeto, subject, que es el nombre genérico para ese fundamento que nos hurta el fenómeno.

Comencemos con la cuestión del sujeto político y la representación.

La crítica de Butler al fundamento tiene una motivación política. El feminismo ha necesitado dotarse imperiosamente de un sujeto político reconocible y estable. Esta necesidad de reconocimiento y de estabilidad va de la mano de una interpretación de la política como un proyecto finalista, atravesado tanto por metas fijadas como por sujetos sustentadores de esas metas. En el caso del feminismo, es el sujeto (sustancia) "mujer" el que ha venido a dar sentido pero también a garantizar la seriedad de los proyectos políticos feministas. Sea como fuere, importa señalar al menos dos cosas de este particular concepto de sujeto. La primera tiene que ver con la representación. El cariz fenomenológico que tiene el sujeto político es que es un sujeto de representación. Esto debe entenderse en toda la ambigüedad del genitivo, pues significa que se trata tando del sujeto que es representado, el sujeto que tiene derechos políticos, como que puede ser él mismo un sujeto que representa y pone a la vista tales derechos, en sí mismo y en otros. Pero la representación no es sólo habilitadora; también es oclusiva y conlleva posibilidades de obliteración. Pensemos en la salida del armario. ¿Consigue la representación para los sujetos gays y lesbianas?, ¿y a qué precio? Por una parte, es claro que la "censura pública" constituye una violencia contra los homosexuales y las lesbianas. Ahora bien, su visibilización como ganancia y como conquista de la representación no deja de estar aquejada por los límites constrictivos de la misma, pues “¿Qué clase de homosexuales y lesbianas debería hacerse visible?” (Butler, 1993b, 311). Al fin y al cabo, la representación es un modo de mostración cerrado y específico. Es, también podría decirse así, la genuina visibilización de la categoría (la representación como la instancia o el caso del concepto), que la hace necesariamente discreccional y restrictiva. Volviendo sobre el ejemplo, no todos los homosexuales pueden acceder a esta representación, no porque no se visibilicen, sino porque la representación impone sus propios límites a la representabilidad, que está estrictamente definida. Así, un transexual podría no convenir a lo que se entiende por representante de los gays (o de las feministas).

Es la misma dificultad que encontramos en la noción jurídica del sujeto. Porque el sujeto político es pensado por el feminismo en términos jurídicos. El sujeto es un sujeto de derechos y la política es el espacio en el que se producen y ganan tales derechos. El sujeto detenta los derechos pero también es precedido y constituido por ellos (siempre que se trate de sujeto y de subjetividad se reproduce la misma ambigüedad, que fue primero señalada por Foucault). Ahora bien, la condición de sujeto de derechos, aun cuando supone que hay un ámbito previo, digamos el derecho, que hace viables a los sujetos que quedan compren- 
didos en él, no termina de hacerse cargo de la verdadera condición productiva y material que tiene todo ámbito de mostración. La representación cumple una función normativa, es una función del derecho, que cumple tanto positiva como negativamente, pero también sirve para sostener y dar entidad a los representados en un modo que trasciende lo jurídico. El derecho conlleva igualmente una particular productividad ontológica, si se nos permite decirlo de esta manera (estamos pensado, lógicamente, en la crítica foucaultiana, de la que Butler se hace eco en varios lugares, a la noción jurídica de poder, por ser insuficiente al dejar fuera las posibilidades habilitadoras que tiene como fuerza constituyente y constrictora a un tiempo). El sujeto "no es una precondición de la política, sino un efecto del poder" (Butler, 2009, iii). Un inmigrante, por ejemplo, no existe hasta tanto no se le otorga alguna carta de naturaleza y el estado echa cuenta con él. Hasta ese momento no es un sujeto de derechos, entre los que está también, claro, el derecho a la vida. Pensemos por ejemplo en las personas antes de cruzar una frontera. La ilegalidad es una forma de cruenta nihilización y lo que tiene lugar en fronteras como la de Melilla es un ejercicio que pretende ser a-jurídico (o extrajurídico, lo mismo que la situación de la prisión de Guantánamo) que demuestra a las claras que en el lugar que precede a la ley no hay, que absolutamente no hay, nada. (En lo que sigue volveremos sobre este ejemplo.)

Pero ser un sujeto en absoluto requiere, primero, la avenencia con ciertas normas que gobiernan el reconocimiento... Y la no-avenencia [con estas normas] pone en cuestión la viabilidad de la vida de uno, las condiciones ontológicas de su persistencia. (Butler, 2009, iv)

Frente a esta noción de sujeto Butler desarrolla la idea de que hay un campo o escenario, que también habrá que entender dramáticamente, en el que los sujetos tienen lugar, take place. La principal objeción a la idea de un sujeto sustantivo es que comprende cualesquiera fenómenos, tanto de su ser como de su acción, como manifestación de una esencia. Como si el interior y el exterior guardaran una relación unívoca, pero también cierta asimetría, que hace que el interior prime sobre el exterior. Por el contrario, el sujeto no es nada más que lo que tiene lugar o acontece, happens, en ese escenario que es el ámbito. El género sólo es real en la medida en que es actuado, performed (Butler, 1990, 278). No hay ni un género, ni tampoco un sexo, que pueda decirse esencial.

La posición del fundamento que elimina la verdad del fenómeno, al convertirlo tan sólo en una apariencia de aquél (digamos en una presencia vicaria), es la metafísica. Butler se refiere reiteradamente a la "metafísica de la sustancia" para describir la ontología que está en la mira de su lectura crítica. Concretamente recuerda que el género es en sí mismo una impugnación a una tal metafísica, toda vez que no exhibe el sexo (que no puede hacer las veces de su solo referente). (En otros textos, cuando habla del lenguaje y de su particular fenomenología, Butler se refiere a esta distancia y a su necesidad como la de una traducción.) ¿Qué es entonces el género? El cuerpo "sólo se conoce por su apariencia generizada" (Butler, 1990, 274). Para la metafísica en el orden del ser hay jerarquía, la cual se traduce fenomenológicamente en la expresión de la esencia, expresión que no es otra cosa que la subordinación del fenómeno a lo que no es fenómeno y queda de parte de la interioridad. Butler propone una "teoría fenomenológica de la constitución" para la que el género es el 
resultado de un acto teatralizado, es decir, de un acto público y ritualizado. ${ }^{1}$ Este es el sentido que tiene en Butler lo performativo, el de actuación, que incorpora el doble sentido de "dramático", pues supone un juego estratégico entre sujetos que actúan, y de "no-refencial", pues la realidad se agota en el acto mismo (Butler, 1990, 273). "los atributos de género no son expresivos sino performativos" (Butler, 1990, 279). El género es el producto de una actuación y nada más que eso. Pero, sobre todo, resulta del todo fenomenológicamente pregnante el que este acto y actividad suprime no sólo toda jerarquía sino aún toda dicotomía. No se trata de que el género sea un juego en el que uno puede asumir e intercambiar las apariencias. El género no es un disfraz ni tampoco un rol. El género es un acto que produce una ficción social, como la llama Butler, a la que pertenece la actuación pública no menos que el yo. En efecto, Butler sugiere

no sólo este yo es un irreparable "afuera" constituido en el discurso social, sino que también la adscripción de la interioridad es ella misma una forma de la fabricación de la esencia, públicamente regulada y sancionada (Butler, 1990, 279).

Ahora bien, esta eventualidad de la acción performativa no significa que el campo no constituya un espacio de determinación. El carácter social del mismo apunta a este hecho. Como recuerda Butler en numerosos textos, se malentiende la performatividad cuando se la vincula con la estabilidad (la persistencia es el modo de la sustancia). Por lo que hace al agente, la actuación no es un ejercicio intencional y atribuible a una voluntad decisoria. "Como acción pública y acto performativo el género no es una elección radical, ni un proyecto que refleja una elección meramente individual, pero tampoco está impuesto o inscrito sobre el individuo" (Butler,1990,277). Simplemente los actores están "siempre ya en el escenario", que es lo único que preexiste: la necesidad de actuar. De hecho, la performatividad de género implica también a "la psique somática" (Butler, 2009, xi). Este rasgo, junto con la condición social del acto, nos aleja todavía más de pensar el género como el resultado de una "decisión deliberada". "Nosotros - dice Butler- no elegimos precisamente los deseos" (Butler, 2009, xii). En la performatividad el sujeto no es soberano. Es "un proceso que implica ser actuado en modo que no siempre comprendemos totalmente" (ibídem). Por su parte, lo obrado performativamente tampoco es estable. Sólo hay un performance que genere una realidad estable, que es lo que Butler llama "performativo divino" (Butler, 2001). Todo lo demás está permanente expuesto a la reelaboración o al fracaso, tanto da.

Como es bien sabido, si la performatividad es vulnerable ello se debe a su condición eventual. La actuación necesita siempre de la repetición. No hay nada estable, ni quien actúa ni lo actuado, pues siempre se está en el trance de la actuación y de la escenificación. La

1 La universalidad, que es el respecto lógico de la esencia, es una universalidad instituida o "universalidad estaturaria” (Butler, 1990). Sobre la crítica de Butler al concepto de universalidad véase J. González Fisac, "Política y feminismo en Judith Butler", en: M.F. Fernández, J.G. Núñez, A. Razquín, J. González y M.N. González, Discurso, compromiso e historia, Barranquilla (Colombia), Ediciones Universidad Simón Bolívar, 2014, p. 125 ss; así como “¿Quién teme a los estudios culturales? La interdisciplinariedad o el problema de la identidad (académica) en Judith Butler", en: A. Mateos-Aparicio y E. De Gregorio (ed.), Identitades en contexto y cultura posmoderna, Oviedo, Kirk Ediciones, 2015, p. 24 ss. 
fuerza del ritual, que es una acción repetible pero también sedimentada en el tiempo, está en el pasado; su debilidad, en el no-ser de la acción futura que, sin embargo, no podemos evitar.

La teoría de la performatividad de género presupone que las normas están actuando sobre nosotros antes siquiera de que tengamos una oportunidad de actuar, y que cuando actuamos recapitulamos las normas que actuan sobre nosotros, quizás en modos inesperados pero todavía vinculados a las normas que nos preceden y exceden. (Butler, 2009, xi)

\section{La crítica de Butler a Arendt y la precariedad del "espacio de aparición"}

Un modo en que puede ser entendido el campo fenomenológico-político que queremos esbozar en Butler está en lo que Arendt llamó "espacio de aparición". En The Human Condition lo define de la siguiente manera:

en el más amplio sentido de la palabra ... el espacio donde yo aparezco ante otros como otros aparecen ante mí, donde los hombres no existen meramente como otras cosas vivas o inanimadas, sino que hacen su aparición de manera explícita (Arendt, 1958, 198-199).

El espacio de aparición constituye una conditio sine qua non para la existencia humana, el espacio en el que las vidas son reconocibles y vivibles, en términos butlerianos. Arendt lo expresa como el hecho de que sólo la presencia de otros hombres garantiza "la realidad del mundo". Estar privado de espacio de aparición es estar privado de realidad, pues la realidad, "humana y políticamente hablando, es lo mismo que la aparición" (Arendt, 1958, 199). El espacio de aparición precede por tanto a la "esfera pública", public realm, que puede organizarse de distintas maneras una vez constituido ese espacio de aparición.

El espacio de aparicion se forma cuando los hombres "están juntos en la manera de discurso y acción", speech and action. Esto, ciertamente, otorga al espacio de aparición cierta carga performativa. Ahora bien, Arendt admite que este espacio no siempre existe y que, cuando existe, tampoco existe para cualesquiera hombres. Butler se refiere a la exclusión, apuntada por Arendt, de mujeres y esclavos. Pero sobre todo es su particular temporalidad (en realidad el dominio de la temporalidad) lo que Butler rechaza, pues el espacio de aparición constituye algo que potencialmente da existencia a la "esfera pública". Esto quiere decir que constituye una estructura o forma definida previamente que distribuye y da o deniega presencia a los hombres. El espacio de aparición estipula no sólo la presencia sino también el modo de participación, la clase de discurso y de acción, que pueden ser visibles en el mismo. Es un espacio codificado y obrado por una codificación que le preexiste. Por eso Arendt entiende que es el resultado del poder, que aquí debe entenderse como dynamis o potentia. El poder precede a lo así constituido por él, que cobra el estatus de actualidad o enérgeia. El espacio de aparición es un espacio normativo pero sobrevenido. Presupone que hay algo así como sujeto, previo al espacio, que puede o no presentarse en el mismo según que se avenga a las condiciones de acción y discurso.

Pues bien, lo que Butler critica de este espacio es que, aun siendo fenomenológico y performativo, no es suficientemente fenomenológico ni performativo. El espacio de aparición se encuentra entre la gente (en el sentido amplio que hemos visto arriba) pero no está 
ligado a la ubicación espacial. Es un espacio esencialmente trasponible. Esta trasponibilidad presupone que es el modo de estar juntos y no sólo el estar juntos en cierto lugar lo que especifica el espacio. Los participantes pueden encontrar su "localización en cualquier tiempo y cualquier lugar" (Arendt, 1958, 198). La política genera un tal espacio y no necesita de él con anterioridad.

Pero el campo de aparición es, además, precario. Tiene una debilidad constitutiva que alcanza también a los que se hacen presentes en él.

Por una parte, la debilidad del campo es la debilidad de toda acción performativa. El hecho de que la acción necesite de la repetición significa que no es nada al margen de su realización. El matrimonio no es sino el acto de casarse, que no es mas que lo que tiene lugar en la ceremonia y en el preciso enunciar de los términos, por quienes pueden hacerlo, etc. (las condiciones fácticas que habilitan la acción y la hacen afortunada, según expresión de Austin, son muchas). La reiteración es necesaria, constituye el ser mismo de la acción performativa, justamente porque no hay norma si no es en el ejercerse mismo de la acción (no hay matrimonio sin casamientos). La reiteración obedece a la posibilidad constitutiva de ruptura o de quiebra de la norma. En efecto, "Cada acto de reproducción corre el riesgo de torcerse o de quedar a la deriva, o de producir efectos que no han sido previstos del todo" (Butler, 2009, iii).

Esto afecta también al derecho y a los sujetos de derecho, esto es, como quienes ejercen aquel, pues el derecho también es una norma que necesita de su ejercicio o ejecución. Así, no se trata tanto de ejerzan su derecho quienes disponen de él previamente como que el derecho mismo emerja y haga su aparición en su ejercicio por parte de sujetos. Butler se ha referido en varios lugares al caso de los inmigrantes que cantan el himno norteamericano en español, demostrando así que son ciudadanos norteamericanos. Si el himno nos identifica, la acción misma de cantarlo, ¿quién puede impedir que su ejecución en español no haga norteamericanos a cualesquiera sujetos que ejerzan esta acción patriótica? En este caso la reiteración de la acción es modificada o reelaborada cumpliendo algunas de sus condiciones, pero sobre todo haciendo buena su condición estrictamente performativa (se es ciudadano al cantar, no se canta porque se sea ciudadano).

Pensemos en el ejercicio de un derecho como el de reunión. El derecho de reunión es un ejercicio precario, necesitado tanto como amenazado por la autorización. Pero la autorización, que podría parecer el ejercicio cabal del derecho, es todo menos inocua. En efecto, si el ejercicio es real, no puede ser simplemente recibido como inteligible o no inteligible para la ley (como autorizado o desautorizado). La ley hace siempre algo más. En realidad, da o priva de realidad a los individuos, porque priva o da realidad a su ejercicio. Porque, ¿qué es un sujeto de derechos sin el ejercicio de sus derechos?

La precariedad performativa del campo estriba además en el hecho de implica alteridad. Butler ha pensado la vulnerabilidad como una "entrega al otro". Hay una dependencia constitutiva que se expresa en la acción performativa. Hemos nacido en un mundo que no controlamos. No se trata de una pédida ocasional; se trata de un control que nunca se tuvo porque siempre hay otros. "estamos desde el comienzo ... entregados a un conjunto primario de otros previo a cualquier individuación” (Butler, 2004, 31).

Butler recuerda a Levinas y a su lectura ética de la otredad en el hecho de la mirada del otro. Cómo sólo podemos reconocernos y ganarnos a nosotros mismos en el rostro del otro, 
toda vez que el nuestro es inasequible. En el mismo sentido, el cuerpo es un punto ciego en el que el sujeto se pierde de alguna manera. En su análisis de la agencia del lenguaje Butler se ha referido a la idea de Joshana Felman de que la relación entre el habla y el cuerpo es una relación "escandalosa", toda vez que es "una relación que consiste en el hecho de que el acto no puede saber lo que está haciendo". El cuerpo, la materia, constituye ese índice de desconocimiento a que nos referimos antes. El acto de habla es siempre un acto corporal de habla en el que uno siempre es desconocedor, hasta cierto punto al menos, de lo que realiza. Siempre habrá algo "de lo que no tiene intención" y sobre el que no ejerce "control" (Butler, 1997, 10).

Por último, completaría este particular mapa de la vulnerabilidad y del campo fenomenológico la vulnerabilidad lingüística, cuando el sujeto es llamado o interpelado, siendo esa llamada, que siempre es una herida, el momento de la constitución de su propia subjetividad. ${ }^{2}$

\section{Bibliografía}

Arendt, Hannah. (1958): The Human Condition, Chicago University Press, Chicago.

Butler, Judith (1990): "Performative Acts and Gender Constitution: An Essay in Phenomenology and Feminist Theory", in: Case, Sue-Ellen: Performing Feminisms: Feminist Critical Theory and Theatre, The John Hopkins University Press, Baltimore, pp. 270-282.

Butler, Judith (1993): "Imitation and Gender Insubordination", in: Abelove, H., Barale, M.A., Halperin, D.M., Lesbian and Gay Studies Reader, Routledge, New York and London, pp. 307-320.

Butler, Judith (1997): Excitable Speech. A politics of the Performative, Routledge, London/ New York.

Butler, Judith (2004): Precarious Life, Verso, London.

Butler, Judith (2009): "Performativity, Precarity and Sexual Politics", AIBR, vol. 4, n 3, pp. i-xiii.

Butler, Judith, Athanasiou, Athena (2013): Dispossesion: The Performative in the Political, Polity Press, Cambridge.

2 Para esto cf. nuestro trabajo "Retórica y fenomenología. Exterioridad y vulnerabilidad del campo del lenguaje", en: Soley-Beltran, P., Sabsay, L. (eds.). Judith Butler en disputa. Barcelona, Egales, 2012. 
